\title{
Tunneling anisotropic thermopower and Seebeck effects in magnetic tunnel junctions
}

\author{
Carlos López-Monís, Alex Matos-Abiague, and Jaroslav Fabian \\ Institut für Theoretische Physik, Universität Regensburg, 93040 Regensburg, Germany \\ (Received 13 September 2013; revised manuscript received 12 September 2014; published 19 November 2014)
}

\begin{abstract}
The tunneling anisotropic magnetothermopower (TAMT) and the tunneling anisotropic spin-Seebeck (TASS) effects are studied for a magnetic tunnel junction (MTJ) composed of a ferromagnetic electrode, a zinc-blende semiconductor, and a normal metal. We develop a theoretical model for describing the dependence of a thermally induced tunneling current across the MTJ on the in-plane orientation of the magnetization in the ferromagnetic layer. The model accounts for the specific Bychkov-Rashba and Dresselhaus spin-orbit interactions present in these systems, which are responsible for the $C_{2 v}$ symmetry we find in the TAMT and the TASS.
\end{abstract}

DOI: 10.1103/PhysRevB.90.174426

PACS number(s): 85.75.-d, 72.25.Mk, 73.50.Jt, 73.50.Lw

\section{INTRODUCTION}

Magnetothermoelectric phenomena have been thoroughly studied for a long time [1,2]. More than twenty years ago Johnson and Silsbee investigated thermoelectric magnetization transport across ferromagnetic-paramagnetic interfaces [3]. However, only during the last lustrum thermoelectric transport has been able to account for the electronic spin degree of freedom [4,5], mainly due to the the discovery of the spin-Seebeck effect [6-8]. This has given birth to the field of spin caloritronics $[9,10]$, which covers the nonequilibrium phenomena related to heat, charge, and spin transport in small magnetic structures.

A three-layer magnetic tunnel junction (MTJ) is a heterostructure consisting of a ferromagnetic conductor, a tunnel barrier, and a ferromagnetic or normal conductor, which are all grown one on top of the other. In the growth direction, the dimension of the MTJ layers is on the nanometric scale; thereby quantum effects have to be considered. Additionally, electric fields, spin voltages and temperature gradients are used to probe their physical properties. These forces create measurable currents that transport charge, spin, and/or heat across the MTJ, making them promising systems for investigating spin caloritronic phenomena [10-18].

Experimentally, it has been observed that the resistance of a MTJ depends on the magnetization orientation with respect to the crystallographic axes of the ferromagnetic layers [19-24]. This effect is known as tunneling anisotropic magnetoresistance (TAMR). Surprisingly, TAMR has also been observed in MTJs with a single magnetic layer [25-27]. However, the behavior of the TAMR varies depending on (i) the specific composition of the MTJ, (ii) whether it has one or two magnetic electrodes, and (iii) whether the magnetization is rotated within a plane perpendicular to the ferromagnetic layer (out-of-plane TAMR) or in the plane of the ferromagnetic layer (in-plane TAMR). MTJs can be made of several kinds of materials. $(\mathrm{Ga}, \mathrm{Mn})$ As magnetic semiconductors are the most commonly used ferromagnetic electrodes [19-23,25]; however TAMR has also been observed in MTJs using Fe (Ref. [26]) and Co (Ref. [27]) or CoFe alloys [24]. Regarding the tunnel barrier, TAMR has been seen with both semiconductor barriers (e.g., GaAs, AlAs, or $\mathrm{ZnSe}$ ) [19-23,26] and insulator barriers such as $\mathrm{Al}_{2} \mathrm{O}_{3}$ or $\mathrm{MgO}$ (Refs. [24,25,27]). For the case of single magnetic layer MTJs, Au is the most commonly used nonmagnetic electrode [25-27]. TAMR phenomena are not restricted to MTJs; they have been observed, for example, in single Co atoms [28]. Recently, anisotropic electric spin injection has also been achieved for ferromagnetic-silicon interfaces [29].

The anisotropy of the tunneling magnetoresistance is due to spin-orbit interaction (SOI) [30]. However, the role SOI plays depends on the specific composition of the MTJ. In $(\mathrm{Ga}, \mathrm{Mn})$ As based MTJs, the origin of the TAMR seems to be the anisotropic density of states (DOS) of the ferromagnetic semiconductor with respect to magnetization, due to its strong SOI in the valence band, combined also with uniaxial strain effects [22,23,25,31]. For MTJs with transition-metal electrodes and an insulating barrier, the Rashba shift of the interface resonant states can produce TAMR [24,32,33], although the anisotropy in the DOS also might induce TAMR [27]. Finally, for MTJs fabricated with transition-metal electrodes and a zinc-blende type semiconductor barrier, TAMR is proposed to be due to interface Bychkov-Rashba (BR) and Dresselhaus (D) SOI [26,34-36].

Recently, an experiment has been performed in the allsemiconductor MTJ (Ga,Mn)As/GaAs/Si:GaAs, in which electric current was driven by a thermal gradient-instead of an electric field-in order to study the in-plane tunneling anisotropic magnetothermopower effect (TAMT) [37]. The thermopower measures the voltage (or current, for the case of closed circuits) induced by a temperature gradient. Hence, for the case of MTJs this quantity is dubbed tunneling magnetothermopower. Naydenova et al. [37] have measured an anisotropic dependence of the thermopower on the magnetization orientation of the ferromagnetic electrode with respect to a reference crystallographic axis. As in the TAMR case, the anisotropy is likely due to the effect of the strong SOI on the DOS of the ferromagnetic semiconductor. Theoretically, the anisotropy induced by SOI has been investigated in the magnetothermopower of a normal-metal/helicalmultiferroic/ferromagnetic MTJ [38] and through ab initio investigations in a $\mathrm{Cu} / \mathrm{Co} / \mathrm{Cu}$ trilayer [39], and more recently in thermogalvanomagnetic transport of ferromagnetic fcc $\mathrm{Co}_{x} \mathrm{Pd}_{1-x}$ alloys from first principles [40].

In this paper, we investigate TAMT and the tunneling anisotropic spin-Seebeck effect (TASS). The former describes the anisotropy of the thermopower (also called Seebeck coefficient), while the latter effect describes the anisotropy of the spin-Seebeck coefficient. We focus on MTJs composed 
of transition-metal electrodes, a zinc-blende semiconductor barrier, and with a single ferromagnetic layer. The calculation is based on the model introduced in Refs. [26,34], which accounts for the BR and D SOIs that are likely to be the cause of the anisotropy for these kind of MTJs. The main result of this work is the characteristic $C_{2 v}$ symmetry found for the TAMS and the TASS, similar to the one observed for the TAMR in the same system [26].

The paper is organized as follows. The theory is presented in Sec. II: The TAMT and the TASS are defined in Sec. II A, the tunneling current is computed in Sec. II B, and the model for describing the MTJ is presented in Sec. II C, namely, the Hamiltonian (Sec. II C 1), the tunneling states (Sec. II C 2), and the transmission probability (Sec. II C 3). The effects of finite temperature are incorporated at a phenomenological level in the model calculations, as explained in Sec. II D. The results are presented in Sec. III, where a phenomenological model is used for describing qualitatively the results (Sec. III A). Finally, a summary is given in Sec. IV.

\section{THEORY}

\section{A. Definitions}

In general, the induced current across a MTJ associated with the spin- $\sigma$ channel, $I_{\sigma}$, is given by the constitutive equation $[41,42]$

$$
\mathbf{I}_{\sigma}=-G_{\sigma}\left(\frac{\Delta \mu_{\sigma}}{e}+S_{\sigma} \Delta T\right),
$$

where $\sigma=\uparrow, \downarrow . G_{\sigma}$ and $S_{\sigma}$ are the conductance and the Seebeck coefficient of the spin- $\sigma$ channel, respectively, $\mu_{\sigma}$ is the spin- $\sigma$ electrochemical potential, and $\Delta T$ an applied thermal gradient. Using Eq. (1) we can write

$$
\begin{aligned}
& \mathbf{I}_{c}=-G\left(\frac{\Delta \mu}{e}+P \frac{\Delta \mu_{s}}{2 e}+S \Delta T\right), \\
& \mathbf{I}_{s}=-G\left(P \frac{\Delta \mu}{e}+\frac{\Delta \mu_{s}}{2 e}+S_{s} \Delta T\right),
\end{aligned}
$$

where $\mathbf{I}_{c / s}=\mathbf{I}_{\uparrow} \pm \mathbf{I}_{\downarrow}$ is the charge/spin current, $\mu=\left(\mu_{\uparrow}+\right.$ $\left.\mu_{\downarrow}\right) / 2$ and $\mu_{s}=\mu_{\uparrow}-\mu_{\downarrow}$ are the charge electrochemical potential and the spin accumulation, respectively, and $P=$ $G_{s} / G$, where $G=G_{\uparrow}+G_{\downarrow}$ is the conductance and $G_{s}=$ $G_{\uparrow}-G_{\downarrow}$ the spin conductance. Furthermore,

$$
S=\frac{1}{G}\left(G_{\uparrow} S_{\uparrow}+G_{\downarrow} S_{\downarrow}\right)
$$

is commonly referred to as the thermopower (or Seebeck coefficient) $[10,43,44]$, and

$$
S_{s}=\frac{1}{G}\left(G_{\uparrow} S_{\uparrow}-G_{\downarrow} S_{\downarrow}\right),
$$

which in the following we shall name the spin-dependent Seebeck coefficient. Additionally, although the quantities

$$
\begin{aligned}
& S_{+}=S_{\uparrow}+S_{\downarrow}, \\
& S_{-}=S_{\uparrow}-S_{\downarrow}
\end{aligned}
$$

appear neither in Eq. (1) nor in Eqs. (2a) and (2b), they as well are relevant material properties. In the literature, $S_{-}$is com-
TABLE I. The material property described by the ratio between an electrochemical potential (spin accumulation) gradient, $\Delta \mu\left(\Delta \mu_{s}\right)$, and a temperature gradient, $\Delta T$, depends on $\Delta \mu_{s}(\Delta \mu), \mathbf{I}_{c}$, and $\mathbf{I}_{s}$. The material properties $S, S_{+}, S_{-}$, and $S_{s}$ are defined in Eqs. (3), (5a), (5b), and (4), respectively. The check mark symbol $(\checkmark)$ means that the corresponding quantity is finite.

\begin{tabular}{cccccc}
\hline \hline Ratio & $\Delta \mu$ & $\Delta \mu_{s}$ & $\mathbf{I}_{c}$ & $\mathbf{I}_{s}$ & Material Property \\
\hline$-\Delta \mu / e \Delta T$ & $\checkmark$ & 0 & 0 & $\checkmark$ & $S$ \\
$-\Delta \mu / e \Delta T$ & $\checkmark$ & $\checkmark$ & 0 & 0 & $S_{+} / 2$ \\
$-\Delta \mu_{s} / 2 e \Delta T$ & $\checkmark$ & $\checkmark$ & 0 & 0 & $S_{-} / 2$ \\
$-\Delta \mu_{s} / 2 e \Delta T$ & 0 & $\checkmark$ & $\checkmark$ & 0 & $S_{s}$ \\
\hline \hline
\end{tabular}

monly referred to as the spin-Seebeck coefficient $[15,38,43-$ 45], while hereinafter we shall refer to $S_{+}$as the effective thermopower.

Table I shows how the measurement of the aforementioned material properties depends on $\Delta \mu, \Delta \mu_{s}, \mathbf{I}_{c}$, and $\mathbf{I}_{s}$. For example, in an open circuit setup $\left(\mathbf{I}_{c}=0\right)$, the ratio $\Delta \mu_{s} / \Delta T$ is related to the spin-Seebeck coefficient $S_{-}$[Eq. (5b), whereas the ratio $\Delta \mu / \Delta T$ will be related either to the thermopower $S$ [Eq. (3)] or to the effective thermopower $S_{+}$[Eq. (5a)], depending on whether there is a finite spin accumulation gradient or not. However, in a closed circuit setup $\left(\mathbf{I}_{c} \neq 0\right)$, Table I shows that the ratio $\Delta \mu_{s} / \Delta T$ is now related to the spin-dependent Seebeck coefficient $S_{S}$ [Eq. (4)].

The tunneling anisotropic magnetothermopower measures the relative dependence of the thermopower [Eq. (3)] or the effective thermopower [Eq. (5a)] on the in-plane magnetization orientation; hence, we have that

$$
\begin{aligned}
\operatorname{TAMT}(\phi) & =\frac{S(0)-S(\phi)}{S(\phi)} \text { or } \\
\operatorname{TAMT}_{[+]}(\phi) & =\frac{S_{+}(0)-S_{+}(\phi)}{S_{+}(\phi)},
\end{aligned}
$$

where $\phi$ is the angle spanned between the magnetization vector and a reference crystallographic axis $[x]$ in the ferromagnet layer (see Fig. 1). Likewise, the tunneling anisotropic spinSeebeck ratio measures the relative dependence of the spinSeebeck coefficient [Eq. (5b)] or the spin-dependent Seebeck coefficient [Eq. (4)] on the in-plane magnetization orientation; hence, we have that

$$
\begin{aligned}
\operatorname{TASS}_{[-]}(\phi) & =\frac{S_{-}(0)-S_{-}(\phi)}{S_{-}(\phi)} \text { or } \\
\operatorname{TASS}(\phi) & =\frac{S_{S}(0)-S_{s}(\phi)}{S_{S}(\phi)} .
\end{aligned}
$$

In the remaining, in order to compute the Seebeck coefficient $S_{\sigma}$ of the spin- $\sigma$ channel, we consider solely a temperature gradient as the driving force responsible for the tunneling current across the MTJ. Thus, Eq. (1) reduces to

$$
\mathbf{I}_{\sigma}=-G_{\sigma} S_{\sigma} \Delta T
$$




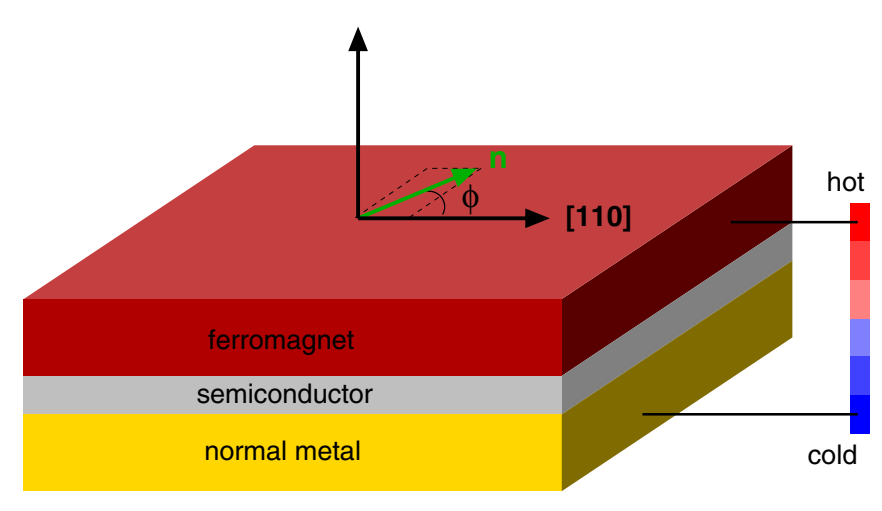

FIG. 1. (Color online) Scheme of a three-layer magnetic tunnel junction. A thermally induced current tunnels across the semiconductor from the ferromagnet electrode into the normal metal. Spin-orbit interaction is responsible for an anisotropic dependence of the (effective) thermopower, and the spin-dependent Seebeck and spin-Seebeck coefficients on the in-plane magnetization orientation $\mathbf{n}$ (green arrow) with respect to a reference crystallographic axis, which in the present case has been taken as the GaAs [110] direction.

\section{B. Tunneling current}

The net current that flows across the MTJ for the spin- $\sigma$ channel is [5]

$$
I_{\sigma}=\frac{1}{e} \int g_{\sigma}(E)\left[f_{L}(E)-f_{R}(E)\right] d E,
$$

where $f_{L}(E) / f_{R}(E)$ is the Fermi-Dirac distribution of the left/right electrode,

$$
g_{\sigma}(E)=\frac{e^{2}}{h} \frac{1}{(2 \pi)^{2}} \int T_{\sigma}\left(\mathbf{k}_{\|}, E\right) d^{2} \mathbf{k}_{\|},
$$

and $T_{\sigma}\left(\mathbf{k}_{\|}, E\right)$ is the transmission probability of the spin- $\sigma$ channel, namely, the probability of an electron with spin- $\sigma$ to tunnel through a potential barrier. The integrals in Eqs. (9) and (10) are performed over the energy, $E$, and the transverse modes, $\mathbf{k}_{\|}$(see next section).

In the linear response regime, the Seebeck coefficient associated with the spin- $\sigma$ channel, $S_{\sigma}$, that appears in Eqs. (3), (4), (5a), and (5b) is

$$
S_{\sigma}=-\frac{1}{G_{\sigma}} \int g_{\sigma}(E)\left(-\frac{\partial f_{0}}{\partial E}\right)\left(\frac{E-\mu_{0}}{e T_{0}}\right) d E,
$$

where

$$
G_{\sigma}=\int g_{\sigma}(E)\left(-\frac{\partial f_{0}}{\partial E}\right) d E
$$

is the conductance of the spin- $\sigma$ channel, and $\mu_{0}$ and $T_{0}$ are the chemical potential and the temperature of the electrodes in equilibrium, respectively (see Appendix A). Notice that all the microscopic information regarding the MTJ is now encoded in the transmission probability $T_{\sigma}$ and the FermiDirac distribution.

\section{Model \\ 1. Hamiltonian}

To study essential effects of anisotropic thermopower and Seebeck effects, we use the model system introduced earlier in Refs. [26,34] to explain TAMR experiments in Fe/GaAs/Au tunnel junctions. This model allows an analytical calculation of the spin-dependent tunneling transmission probability $T_{\sigma}$ in the presence of SOI and, in our view, can serve as a benchmark for analyzing TAMT and TASS effects.

The structure we model is shown Fig. 1. The metallic layers are described as free and independent electron gases [46] in a semi-infinite space (meaning that the only boundaries are the interfaces between the electrodes and the semiconductor layer). A more sophisticated description is given for the GaAs layer, where the extended Kane model is used [5]. This model accounts for the bulk inversion asymmetry of the GaAs semiconductor (due to its zinc-blende crystal structure) and the structure inversion asymmetry of the MTJ. The former asymmetry causes a D-SOI field, while the latter one causes a BR-SOI field. The combination of both spinorbit fields leads to an overall anisotropic SOI with the required $C_{2 v}$ symmetry observed in TAMR experiments using a Fe/GaAs/Au MTJ [26]. Ab initio calculations have confirmed that the origin of this symmetry is the atomic structure at both interfaces of the MTJ $[47,48]$.

An external magnetic field, $\mathbf{B}_{\text {ext }}$, is used to control the magnetization orientation of the ferromagnetic electrode. This occurs in the saturation limit, where the strength of the magnetic field is such that the magnetization is forced to remain parallel to $\mathbf{B}_{\text {ext }}$. However, the Zeeman splittings due to $\mathbf{B}_{\text {ext }}$ are negligible compared to the exchange energy in the ferromagnet. Furthermore, the orbital effects due to $\mathbf{B}_{\mathrm{ext}}$ can be safely neglected as long as the magnetization remains in-plane [36].

We use two different models to describe the tunneling barrier, namely, a rectangular potential barrier and a Diracdelta function potential barrier (Fig. 2). Both models have been successful in describing TAMR experiments [26], while the latter one has been used for describing TAMT experiments as well [37]. A version of the rectangular barrier model in the absence of SOI has recently been applied to the theoretical

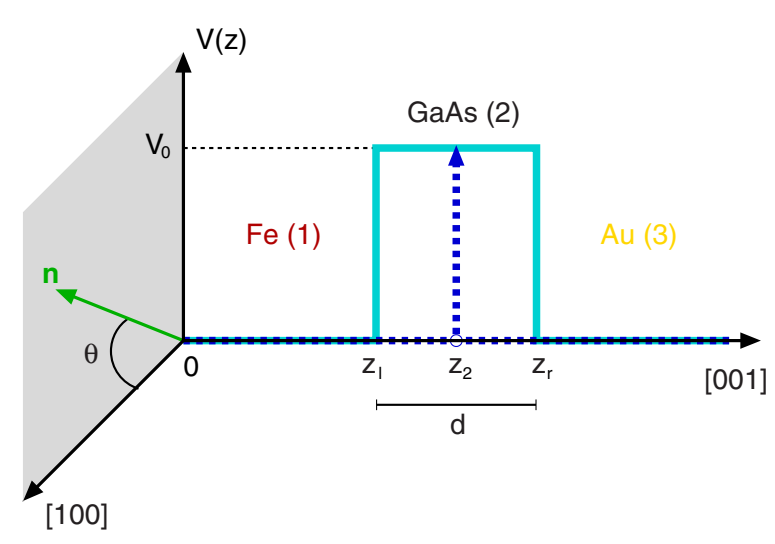

FIG. 2. (Color online) Schematics of the two models used for describing the tunneling barrier (Table II). The solid (dashed) light (dark) blue line represents the rectangular barrier (delta function) model, where $d$ and $V_{0}$ correspond to the thickness and the height of the GaAs layer, respectively. Additionally, the figure shows the relative orientation $\theta$ of the in-plane magnetization $\mathbf{n}$ (green arrow) with respect to the [100] crystallographic axis of the GaAs layer. 
TABLE II. The rectangular barrier and delta function models. The subscripts 1, 2, and 3 correspond to the Fe, GaAs, and Au layers, respectively, and in the rectangular barrier model $l$ and $r$ refer to the left and right interfaces (Fig. 2). The magnetization direction is given by the unit vector $\mathbf{n}$, the exchange energy by the parameter $\Delta$, and $\theta(z)$ is the Heaviside step function. The Bychkov-Rashba coupling strength at the $\mathrm{Fe} / \mathrm{GaAs}$ and GaAs/Au interfaces are given by the parameters $\alpha_{l}$ and $\alpha_{r}$, respectively, and $\delta(z)$ is the Dirac-delta function. The bulk Dresselhaus coupling strength is $\gamma$, and $\bar{\gamma}$ is the linearized Dresselhaus parameter [34]. The parameter $\alpha$ describes the BR SOI in the Dirac-delta function barrier limit.

\begin{tabular}{lcr}
\hline \hline & Rectangular Potential Barrier & Dirac-delta Function Potential Barrier \\
\hline$m(z)$ & $m_{1} \theta\left(-z+z_{l}\right)+m_{2} \theta\left(z-z_{l}\right)+\left(m_{3}-m_{2}\right) \theta\left(z-z_{r}\right)$ & $m_{1} \theta\left(-z+z_{2}\right)+m_{3} \theta\left(z-z_{2}\right)$ \\
$\mathcal{V}(z)$ & $V_{0} \mathcal{I}\left[\theta\left(z-z_{l}\right)-\theta\left(z-z_{r}\right)\right]$ & $\left(V_{0} d\right) \mathcal{I} \delta\left(z-z_{2}\right)$ \\
$\mathbf{H}(z)$ & $-\Delta \mathbf{n} \theta\left(-z+z_{l}\right)$ & $-\Delta \mathbf{n} \theta\left(-z+z_{2}\right)$ \\
$\mathcal{V}_{B R}$ & $\frac{1}{\hbar}\left(p_{x} \sigma_{y}-p_{y} \sigma_{x}\right)\left[\alpha_{l} \delta\left(z-z_{l}\right)-\alpha_{r} \delta\left(z-z_{r}\right)\right]$ & $\frac{\alpha}{\hbar}\left(p_{x} \sigma_{y}-p_{y} \sigma_{x}\right) \delta\left(z-z_{2}\right)$ \\
$\mathcal{V}_{D}$ & $\frac{1}{\hbar^{3}} \gamma\left(p_{x} \sigma_{x}-p_{y} \sigma_{y}\right) p_{z}\left[\theta\left(z-z_{l}\right)-\theta\left(z-z_{r}\right)\right] p_{z}$ & $\frac{\bar{\gamma}}{\hbar}\left(p_{x} \sigma_{x}-p_{y} \sigma_{y}\right) \delta\left(z-z_{2}\right)$ \\
\hline \hline
\end{tabular}

investigation of the tunneling magnetothermopower in MTJs with two magnetic electrodes [49]. We shall next discuss the Hamiltonian for the system and, in the following, the differences between both models will be indicated in Table II.

With all this in mind, the Hamiltonian we use for describing the MTJ is

$$
\mathcal{H}=\mathcal{T}+\mathcal{V}+\mathcal{V}_{\mathcal{Z}}+\mathcal{V}_{\mathcal{B R}}+\mathcal{V}_{\mathcal{D}}
$$

Since the MTJ is a heterostructure, the effective mass of the electrons is, in general, different in each layer (although we take it to be constant within each one), meaning the mass becomes position dependent, and so does the kinetic energy operator $\mathcal{T}[50]$,

$$
\mathcal{T}(z)=\mathbf{p} \cdot\left[\frac{1}{2 m(z)} \mathbf{p}\right] \mathcal{I},
$$

where $\mathbf{p}=-i \hbar \Delta$ is the momentum operator, $m(z)$ is the position-dependent effective mass (see Table II), and $\mathcal{I}$ is the unit matrix in spinor space. The second term in Eq. (13), $\mathcal{V}$, describes the semiconductor tunneling barrier (see Table II). The third term, $\mathcal{V}_{\mathcal{Z}}$, accounts for the exchange energy due to the magnetization in the ferromagnetic lead (Stoner model) [51],

$$
\mathcal{V}_{\mathcal{Z}}(z)=\frac{1}{\hbar} \mathbf{H}(z) \cdot \mathbf{S}
$$

$\mathbf{H}(z)$ is the effective exchange field (see Table II), $\mathbf{S}=(\hbar / 2) \boldsymbol{\sigma}$ is the spin operator, and $\sigma=\left(\sigma_{x}, \sigma_{y}, \sigma_{z}\right)$ are the Pauli matrices. $\mathbf{H}(z)$ is taken to be in-plane, meaning $\mathbf{n}=(\cos \theta, \sin \theta, 0)$, where $\theta$ defines the angle between the magnetization and the GaAs [100] crystallographic axis. In previous experiments with $\mathrm{Fe} / \mathrm{GaAs} / \mathrm{Au}$ MTJs [26], the reference axis was taken as the GaAs [110] direction. Therefore, we prefer to express the magnetization direction relative to the [110] axis by introducing the angle shifting $\phi=\theta-\pi / 4$. Finally, the terms $\mathcal{V}_{B R}$ and $\mathcal{V}_{D}$ in Eq. (13) account for the BR-SOI and the D-SOI, respectively (see Table II).

\section{Tunneling states}

The wave functions describing the conduction electrons are obtained by solving the stationary Pauli-Schrödinger equation

$$
\mathcal{H} \boldsymbol{\psi}(\mathbf{R})=E \boldsymbol{\psi}(\mathbf{R}),
$$

for the Hamiltonian given in Eq. (13), where

$$
\boldsymbol{\psi}(\mathbf{R})=\left(\begin{array}{c}
\psi_{\uparrow}(\mathbf{R}) \\
\psi_{\downarrow}(\mathbf{R})
\end{array}\right)
$$

is a spinor which components correspond to the wave functions for spin-up and spin-down electrons, respectively, and $\mathbf{R}=$ $(x, y, z)$. The solutions of Eq. (16) are the eigenenergies, $E$, and eigenstates of the system. Since the transversal modes, $\mathbf{p}_{\|}$, are conserved,

$$
\psi_{\sigma}(\mathbf{R})=e^{i \mathbf{k}_{\|} \cdot \mathbf{r}} \varphi_{\sigma}(z),
$$

where $\varphi_{\sigma}(z)$ is a solution of the Pauli-Schrödinger equation for the longitudinal modes, $\mathbf{r}=(x, y), \mathbf{k}_{\|}=\left(k_{x}, k_{y}\right)$, and $\mathbf{p}_{\|}=$ $\hbar \mathbf{k}_{\|}$. Notice that the longitudinal (out-of-plane) modes have decoupled from the transverse (in-plane) modes. Replacing Eq. (18) in Eq. (16) allows us to solve analytically the resulting one-dimensional stationary Pauli-Schrödinger equation (see Appendices $\mathrm{C}$ and D). We find that

$$
\begin{aligned}
& \boldsymbol{\varphi}_{i}(z)=\frac{1}{\sqrt{k_{1 \sigma}}} e^{i k_{1 \sigma} z} \boldsymbol{v}_{\sigma}, \\
& \boldsymbol{\varphi}_{t}(z)=\left(t_{\sigma, \sigma} \boldsymbol{v}_{\sigma}+t_{\bar{\sigma}, \sigma} \boldsymbol{v}_{\bar{\sigma}}\right) e^{i k_{3} z},
\end{aligned}
$$

where

$$
\begin{aligned}
k_{1 \sigma} & =\sqrt{\frac{2 m_{1} E}{\hbar^{2}}+k_{F \sigma}^{2}-k_{\|}^{2}}, \\
k_{3} & =\sqrt{\frac{2 m_{3} E}{\hbar^{2}}+\kappa_{F}^{2}-k_{\|}^{2}},
\end{aligned}
$$

and

$$
\boldsymbol{v}_{\sigma}=\frac{1}{\sqrt{2}}\left(\begin{array}{c}
1 \\
\sigma e^{i \theta}
\end{array}\right),
$$

where $\sigma=\uparrow(1), \downarrow(-1), E$ is the energy measured from the Fermi level, $k_{F \sigma}$ is the Fermi wave vector amplitude corresponding to the spin- $\sigma$ channel in the magnetic electrode, and $\kappa_{F}$ is the Fermi wave vector amplitude in the normal-metal region.

The subscripts $i$ and $t$ in Eqs. (19a) and (19b) stand for the incident and the transmitted wave functions, respectively. The coefficient $t_{\sigma, \sigma} / t_{\bar{\sigma}, \sigma}$ represents the transmission probability amplitude for a tunneling process in which the electron spin is preserved/flipped. These amplitudes are computed analytically by solving the set of linear equations obtained when 
imposing the boundary conditions to the wave functions (19a) and (19b). The expressions obtained for $t_{\sigma, \sigma} / t_{\bar{\sigma}, \sigma}$ within the rectangular barrier and the delta function models are given in Eq. (C11a)/(C11b) (Appendix C) and Eq. (D5a)/(D5b) (Appendix D), respectively.

\section{Transmission probability}

In general, the transmission probability is defined as

$$
T=\frac{J_{z}^{(t)}}{J_{z}^{(i)}},
$$

where $J_{z}^{(i)}$ and $J_{z}^{(t)}$ are the incident and transmitted probability current densities across the MTJ, respectively. The probability current density is given by the expression

$$
J_{z}(z)=\frac{e \hbar}{2 i m(z)}\left[\psi^{\dagger} \frac{\partial}{\partial z} \psi-\left(\psi^{\dagger} \frac{\partial}{\partial z} \psi\right)^{*}\right]
$$

Therefore, using the wave functions for the incident and transmitted electrons computed in the previous section [Eqs. (19a) and (19b)], we find that the corresponding current probability densities are

$$
\begin{aligned}
& J_{z}^{(i)}=-\frac{\hbar e}{m_{1}}, \\
& J_{z}^{(t)}=-\frac{\hbar e}{m_{3}} k_{3}\left(\left|t_{\sigma, \sigma}\right|^{2}+\left|t_{\bar{\sigma}, \sigma}\right|^{2}\right) .
\end{aligned}
$$

Finally, the transmission probability for a spin- $\sigma$ incoming electron is found by replacing Eqs. (24a) and (24b) in Eq. (22),

$$
T_{\sigma}\left(E, \mathbf{k}_{\|}\right)=\frac{m_{1}}{m_{3}} k_{3}\left(\left|t_{\sigma, \sigma}\right|^{2}+\left|t_{\bar{\sigma}, \sigma}\right|^{2}\right) .
$$

\section{Temperature effects}

With the aim to investigate finite-temperature effects on the TAMT, TAMT $_{[+]}$, TASS $_{[-]}$, and TASS (see Sec. II A), we include the temperature dependence of the relevant system parameters.

We assume that the temperature dependence of the spin polarization of the density of states in the magnetic region is similar to Bloch's law, i.e.,

$$
P_{g}(T)=P_{g}(0)\left(1-\eta T^{3 / 2}\right),
$$

where

$$
P_{g}(0)=\frac{k_{F \uparrow}-k_{F \downarrow}}{k_{F \uparrow}+k_{F \downarrow}}
$$

is the spin polarization of the density of states at zero temperature, $\eta$ is a phenomenological parameter to be extracted from experimental data, and $k_{F \uparrow}$ and $k_{F \downarrow}$ are the Fermi wave vectors of the spin-up and spin-down channels, respectively. An assumption similar to that in Eq. (26) has been successfully used for the phenomenological description of the temperature dependence of the TMR effect in MTJs [52,53].

In order to account for the temperature dependence of the spin polarization in the magnetic electrode we introduce effective, temperature-dependent wave vectors,

$$
k_{\mu \sigma}=\sqrt{\frac{2 m_{1} \mu_{\sigma}}{\hbar^{2}}},
$$

where $\mu_{\sigma}$ is the chemical potential corresponding to the spin- $\sigma$ channel in the magnetic lead. The generalization of Eq. (27) then reads as

$$
P_{g}(T)=\frac{k_{\mu \uparrow}(T)-k_{\mu \downarrow}(T)}{k_{\mu \uparrow}(T)+k_{\mu \downarrow}(T)} .
$$

For temperatures such that $k_{B} T \ll \mu$, the particle density can be approximated as

$$
n(T) \approx \frac{1}{6 \pi^{2}}\left(k_{\mu \uparrow}^{3}+k_{\mu \downarrow}^{3}\right) .
$$

Combining the equations above and taking into account the conservation of the number of particles [i.e., $n(0)=n(T)$ ], we find

$$
k_{\mu \sigma}(T)=\left[\frac{k_{F \uparrow}^{3}+k_{F \downarrow}^{3}}{2+6 P_{g}^{2}(T)}\right]^{1 / 3}\left[1+\sigma P_{g}(T)\right] .
$$

Thus, by replacing the Fermi wave vectors $k_{F \sigma}$ by the effective, temperature-dependent ones, $k_{\mu \sigma}(T)$, we can introduce the temperature dependence of the spin polarization $P(T)$. Such a dependence is characterized by the parameter $\eta$, which we extract by fitting the experimental data of the temperature dependence of the TAMR (see Ref. [54]) to our theory.

The temperature also influences the size of the energy gap in the semiconducting region and, therefore, the height of the tunneling barrier too. The temperature dependence of the band gap can be estimated by using Varshni's empirical relation $[55,56]$,

$$
E_{g}(T)=E_{g}(0)-\frac{a T^{2}}{T+b},
$$

where $a$ and $b$ are system parameters, which for the case of GaAs, for example, take the values $a=5.5 \times 10^{-4} \mathrm{eV} / \mathrm{K}$ and $b=225 \mathrm{~K}$ [56]. For the case of an Fe/GaAs/Au MTJ the height of the barrier is about half the GaAs fundamental gap. Therefore the temperature dependence of the tunneling barrier height is determined by

$$
V_{0}(T)=V_{0}-\frac{a T^{2}}{2(T+b)} .
$$

Here $V_{0}$ represents the height of the barrier at zero temperature.

The BR and D SOI parameters depend relatively weakly on the temperature $[5,57]$ and we, therefore, neglect the temperature effects on such parameters.

\section{RESULTS}

Here we investigate the behavior of the coefficients TAMT, $\mathrm{TAMT}_{[+]}$, TASS, and $\mathrm{TASS}_{[-]}$(see Sec. II A) for both the thin-barrier limit for which we use the Dirac-delta function barrier model and the thick-barrier limit which is treated within the rectangular barrier approach. The calculations were performed for an $\mathrm{Fe} / \mathrm{GaAs} / \mathrm{Au}$ tunnel junction. For such a system, the height of the semiconducting barrier is $V_{0}=$ $0.75 \mathrm{eV}$. The Fermi momenta in Fe and $\mathrm{Au}$ are $k_{F \uparrow}=1.05 \times$ $10^{8} \mathrm{~cm}^{-1}, k_{F \downarrow}=0.44 \times 10^{8} \mathrm{~cm}^{-1}$, and $\kappa_{F}=1.2 \times 10^{8} \mathrm{~cm}^{-1}$, respectively. The electron effective mass in GaAs was taken as $m_{2}=0.067 m_{0}$, while for the metallic regions the bare electron 
mass $m_{0}$ was assumed. For the Dresselhaus SOI parameter in the GaAs barrier we used the value $\gamma=24 \mathrm{eV} \AA^{3}$ [5].

Previous calculations have shown that for the description of the TAMR only the BR-SOI at the Fe/GaAs interface is relevant [34]. For the sake of simplicity we first adopt such an approximation by setting $\alpha_{r}=0$ and $\alpha_{l}=\alpha$. The effects of finite $\alpha_{r}$ will be considered later on in this section. The calculations discussed below were done for two different values of the $\mathrm{BR}$ parameter corresponding to the Fe/GaAs interface, $\alpha=33.52 \mathrm{eV} \AA^{2}$ and $\alpha=-23.35 \mathrm{eV} \AA^{2}$. These values were obtained by fitting the experimental data of the TAMR measured in an Fe/GaAs/Au MTJ at $T=4 \mathrm{~K}$ [54]. Furthermore, by fitting the experimentally measured temperature dependence of the TAMR we obtained the values $\eta=1.5 \times 10^{-4} \mathrm{~K}^{-3 / 2}$ and $\eta=0.66 \times 10^{-4} \mathrm{~K}^{-3 / 2}$ for the phenomenological parameter introduced in Sec. II D. The two values of the $\alpha$ and $\eta$ parameters correspond to the experimentally used bias voltages, $-90 \mathrm{mV}$ and $90 \mathrm{mV}$, respectively. For the Dirac-delta function barrier model we took the effective barrier height as $V_{0} d$ with $d=20 \AA$. The linearized Dresselhaus parameter was estimated as $\bar{\gamma}=\gamma q_{0}$, with $q_{0}=\sqrt{2 m_{2} V_{0} / \hbar^{2}}$.

The left and right panels of Fig. 3 show, respectively, the TAMT [Eq. (6a)] and the TAMT $[+][$ Eq. (6b)] as functions of the magnetization orientation. The upper panels [(a) and (b)] correspond to the Dirac-delta function barrier approach while the lower ones [(c) and (d)] show the results of the rectangular barrier model. The solid (dashed) lines were obtained by using the values $\alpha=33.52 \mathrm{eV} \AA^{2}\left(\alpha=-23.35 \mathrm{eV} \AA^{2}\right)$ and $\eta=1.5 \times 10^{-4} \mathrm{~K}^{-3 / 2}\left(\eta=0.66 \times 10^{-4} \mathrm{~K}^{-3 / 2}\right)$. Similar calculations of the angular dependence of the TASS T-] $_{[\text {Eq. (7a)] }}$ and the TASS [Eq. (7b)] are shown in Fig. 4. As can be seen,
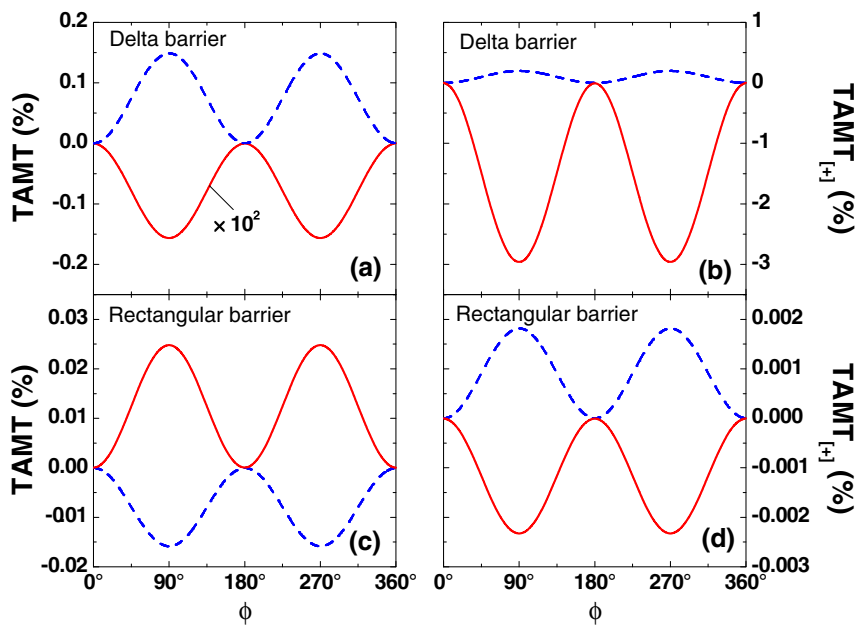

FIG. 3. (Color online) Tunneling anisotropic magnetothermopower [Eqs. (6a) and (6b)] dependence on the angle $\phi$ spanned between the magnetization and the GaAs [110] direction. (a), (b) Results of the Dirac-delta model. (c), (d) Results of the rectangular barrier model. Solid (dashed) lines were computed by using the values $\alpha=33.52 \mathrm{eV} \AA^{2}\left(\alpha=-23.35 \mathrm{eV} \AA^{2}\right)$ and $\eta=1.5 \times 10^{-4} \mathrm{~K}^{-3 / 2}$ $\left(\eta=0.66 \times 10^{-4} \mathrm{~K}^{-3 / 2}\right)$. The average temperature between left and right leads has been set to the value $T=4 \mathrm{~K}$ and the width of the rectangular barrier is $d=80 \AA$.
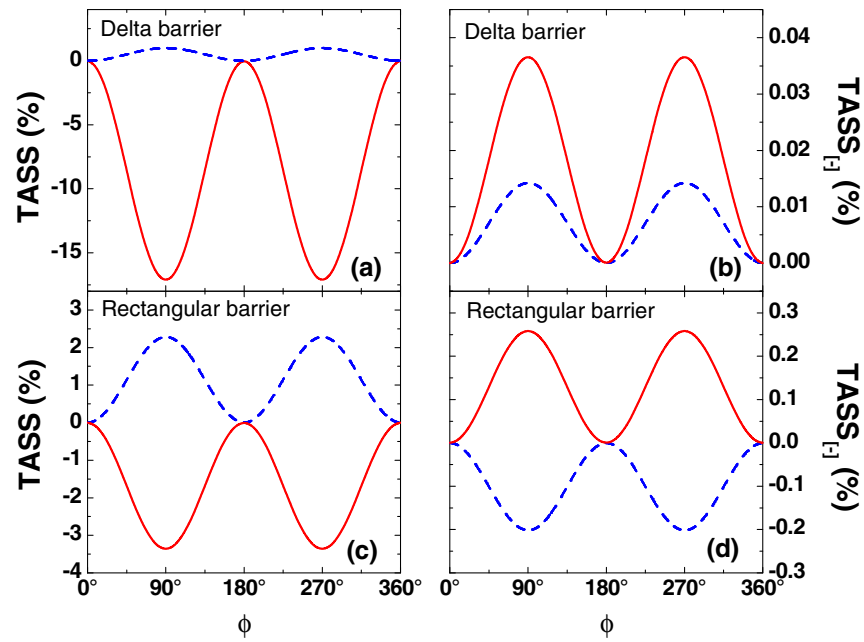

FIG. 4. (Color online) Tunneling anisotropic spin-Seebeck [Eqs. (7a) and (7b)] dependence on the angle $\phi$ spanned between the magnetization and the GaAs [110] crystallographic axis. Description as in Fig. 3.

all the curves in Figs. 3 and 4 exhibit a twofold symmetry with respect to the magnetization orientation. Such a symmetry is a signature of the $C_{2 v}$ symmetry of the Fe/GaAs interface.

By comparing the curves in Figs. 3(a) and 3(c) one can conclude that the size of the TAMT strongly changes when going from the thin to the thick barrier limits. The changes in the amplitude range from one to three orders of magnitude. A similar behavior is obtained for the TAMT ${ }_{[+]}$[see Figs. 3(b) and 3(d)]. Furthermore, the signs of both the TAMT and $\mathrm{TAMT}_{[+]}$change when the sign of the BR SOI is inverted. However, while for a given value of $\alpha$ the sign of the TAMT changes also when going from the thin to the thick barrier limit, the sign of the $\mathrm{TAMT}_{[+]}$remains the same. The TAMT and $\mathrm{TAMT}_{[+]}$differ also quantitatively; the former is, for both the Dirac-delta function and rectangular barriers, about one order of magnitude larger. It is worth remarking that, in general, one cannot expect that apart from the symmetry properties, the TAMT and TAMT $\mathrm{T}_{[+]}$exhibit a similar behavior. After all, these quantities obey different definitions [Eqs. (6a) and (6b)] and different physical situations (see Table I). The same applies for the TASS and $\operatorname{TASS}_{[-]}$, whose magnetization orientation dependence is shown in Fig. 4.

As for the TAMT and $\mathrm{TAMT}_{[+]}$, the TASS and $\mathrm{TASS}_{[-]}$ amplitudes obtained in the thin and thick barrier limits appear to be different (see Fig. 4), although in this case the difference is not three but one order of magnitude. The large differences between the results in the thin and thick barrier limits originate from the intrinsically different nature of the energy dependence of the transmissivity function, which within the Dirac-delta function model exhibits a power-law dependence while for the rectangular barrier is dominated by an exponential behavior. Further differences are introduced by the distinct levels of approximation of the Dresselhaus SOI, which is linear in momentum in the Dirac-delta function barrier model but cubic in the rectangular barrier approximation (see Table II).

The temperature dependence of the amplitude of the TAMT (left panel) and TAMT $_{[+]}$(right panel) is shown in Fig. 5. 

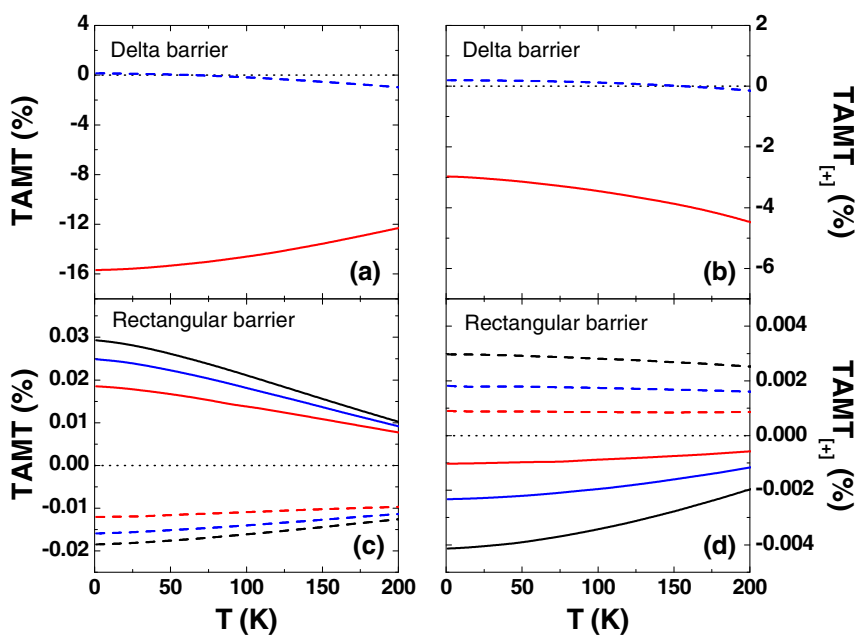

FIG. 5. (Color online) Amplitude of the tunneling anisotropic magnetothermopower [Eqs. (6a) and (6b)] as a function of temperature for magnetization oriented along the [110] crystallographic direction $\left(\phi=90^{\circ}\right)$. (a), (b) Results of the Dirac-delta model. (c), (d) Results of the rectangular barrier model. Solid (dashed) lines were computed by using the values $\alpha=33.52 \mathrm{eV} \AA^{2}\left(\alpha=-23.35 \mathrm{eV} \AA^{2}\right)$, and $\eta=1.5 \times 10^{-4} \mathrm{~K}^{-3 / 2}\left(\eta=0.66 \times 10^{-4} \mathrm{~K}^{-3 / 2}\right)$. In (c) and (d) the pairs of solid and dashed lines with the largest, middle, and smallest absolute values of the TAMT and $\mathrm{TAMT}_{[+]}$at $T=0 \mathrm{~K}$ correspond to barrier thicknesses $d=60,80,120 \AA$, respectively.

Solid and dashed lines correspond to two different sets of $\alpha$ and $\eta$ parameters. In the temperature range considered, the amplitude of the TAMR was experimentally found to decrease with temperature [54]. Like the TAMR, the absolute value of the amplitudes of the TAMT, $\mathrm{TAMT}_{[+]}$, and TASS $_{[-]}$computed for the rectangular barrier model [see Figs. 5(c), 5(d), and Fig. 6(d), respectively] decrease with temperature. However, the other curves shown in Figs. 5(a), 5(b), and Figs. 6(a), 6(b), and 6(c) do not, in general, exhibit such a trend in their temperature dependence. This reveals that the temperature dependence of the Seebeck and spin-Seebeck related coefficients is more complex than the one measured in the TAMR experiments. The decreasing of TAMR with temperature is a consequence of the fact that the anisotropy of the conductance decreases with the spin polarization, which in turn decreases with temperature. However, the Seebeck coefficient related to the spin- $\sigma$ channel, $S_{\sigma}$, does not depend only on the conductance but rather on the ratio between the integral, $\int g_{\sigma}(E)\left[-\partial f_{0}(E)\right]\left(E-\mu_{0}\right) d E$, and the conductance [see Eq. (11)]. The anisotropy of both the integral and the conductance decrease with the spin polarization and, therefore, with temperature. However, the temperature dependence of the anisotropy of the ratio $S_{\sigma}$ is determined by the competition between the decreasing rates of the integral and the conductance. As a consequence the anisotropy of the Seebeck and spin-Seebeck related ratios do not necessary decrease with temperature over all the temperature range. At higher temperatures, where our simplified model calculations are no longer valid, the anisotropy should again decrease and eventually vanish when the temperature is higher than the Curie temperature of the magnetic lead. Thus, our results suggest that the anisotropy of the Seebeck and spin-Seebeck
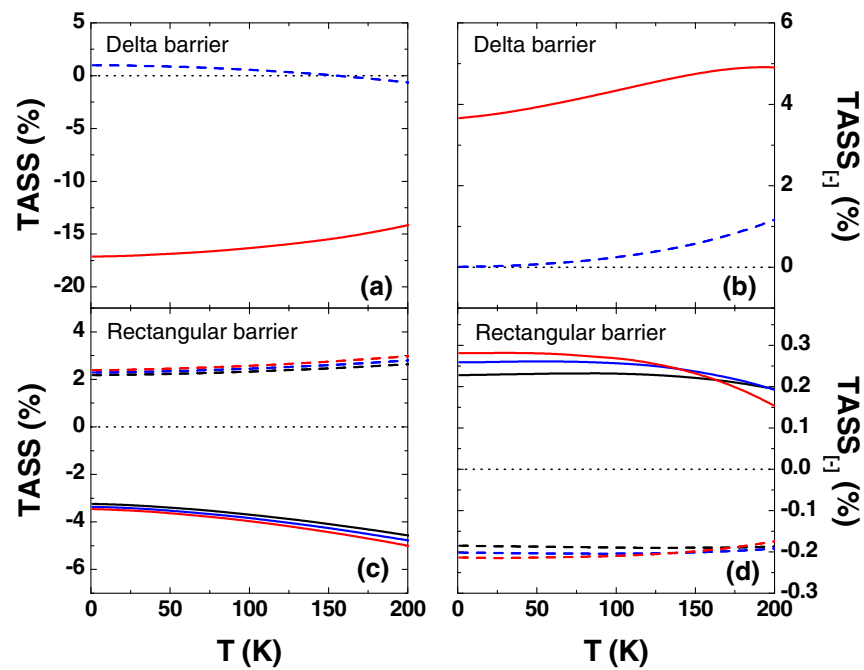

FIG. 6. (Color online) Amplitude of the tunneling anisotropic spin-Seebeck ratio [Eqs. (7b) and (7a)] as a function of temperature for magnetization oriented along the [110] crystallographic direction $\left(\phi=90^{\circ}\right)$. (a), (b) Results of the Dirac-delta model. (c), (d) Results of the rectangular barrier model. Solid (dashed) lines were computed by using the values $\alpha=33.52 \mathrm{eV} \AA^{2}\left(\alpha=-23.35 \mathrm{eV}^{2}\right)$ and $\eta=1.5 \times 10^{-4} \mathrm{~K}^{-3 / 2}\left(\eta=0.66 \times 10^{-4} \mathrm{~K}^{-3 / 2}\right)$. In (c) and (d) the pairs of solid and dashed lines with the smallest, middle, and largest absolute values of the TASS and TASS $_{[-]}$at $T=0 \mathrm{~K}$ correspond to barrier thicknesses $d=60,80,120 \AA$, respectively.

related coefficients may exhibit a nonmonotonic temperature dependence.

As shown in Figs. 5(c) and 5(c), in the temperature range investigated, the absolute value of both the TAMT and TAMT $_{[+]}$increase as the thickness of the barrier decreases. An opposite behavior is found for the TASS and the $\operatorname{TASS}_{[-]}$(at least up to $T \approx 150 \mathrm{~K}$, where the $\mathrm{TASS}_{[-]}$inverts its behavior), as displayed in Figs. 6(c) and 6(c), respectively.

In order to investigate the possible effects of the BR-SOI at the GaAs/Au interface (which until now have been neglected) we have computed the dependence of the amplitudes of the TAMT and TASS (see Fig. 7) and the TAMT $[+]$ and TASS $_{[-]}$ (see Fig. 8) as functions of both the BR-SOI parameters of the $\mathrm{Fe} / \mathrm{GaAs}$ and the GaAs/Au interfaces (i.e., $\alpha_{l}$ and $\alpha_{r}$, respectively).

According to Fig. 7, the amplitudes of both the TAMT and the TASS depend very weakly on $\alpha_{r}$ and are dominated by the BR-SOI at the $\mathrm{Fe} / \mathrm{GaAs}$ interface (i.e., by $\alpha_{l}$ ). A similar behavior was previously found for the TAMR [34]. For the cases of the TAMT $_{[+]}$and TASS $[-]$(see Fig. 8) the dependence on $\alpha_{r}$ appears to be stronger than in the TAMT and TASS cases. Nevertheless, while switching off the BR SOI at the $\mathrm{GaAs} / \mathrm{Au}$ interface (i.e., setting $\alpha_{r}=0$ ) does not necessarily lead to vanishing $\mathrm{TAMT}_{[+]}$and $\mathrm{TASS}_{[-]}$, the anisotropic effects vanish when $\alpha_{l}=0$. Therefore, the SOI parameter at the $\mathrm{Fe} / \mathrm{GaAs}\left(\alpha_{l}\right)$ together with the D SOI strength $(\gamma)$ can still be regarded as the physically crucial parameters for the anisotropic effects.

Finally, it is worth remarking that the values of the amplitudes of the TAMT, TAMT $_{[+]}$, TASS, and TASS $_{[-]}$predicted by our calculations are all within current experimentally 

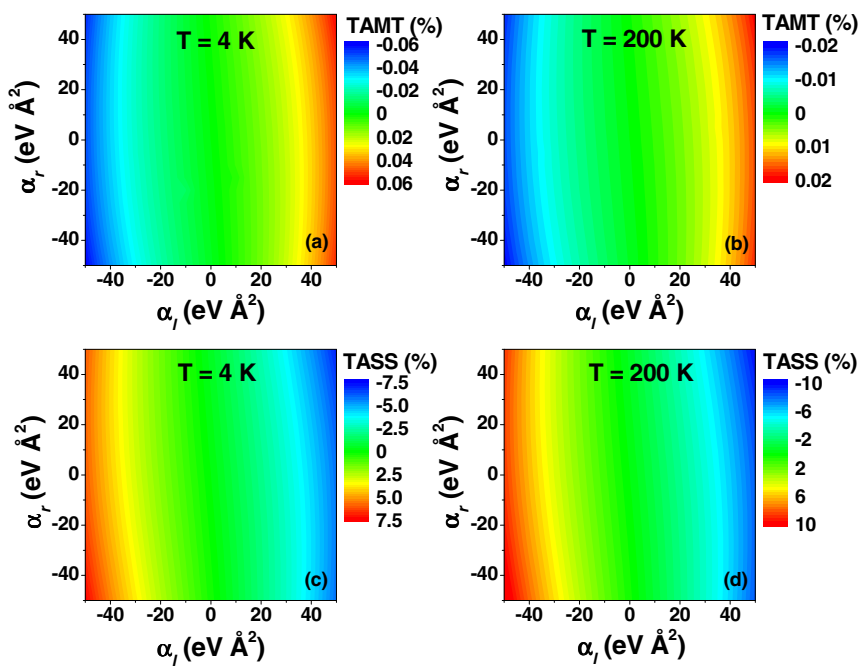

FIG. 7. (Color online) Amplitudes of the TAMT [(a) and (b)] and the TASS [(c) and (d)] as functions of the BR-SOI parameters $\alpha_{l}$ and $\alpha_{r}$. The results correspond to an $80 \AA$ thick rectangular barrier.

accessible resolution. Even though the amplitudes of the TAMT and $\mathrm{TAMT}_{[+]}$are seemingly small [see, for example, Figs. 5(c) and 5(d)], the corresponding thermovoltages that lead to those values are, in principle, still measurable. Typically, in these systems the thermovoltages measured are in the range of hundreds of $\mu \mathrm{V}$ [37]; hence, taking this as a reference, an anisotropy of the order of $10^{-3} \%$ can be detected by measuring the thermovoltage with a resolution of the order of $n V$.

\section{A. Phenomenological model}

Inspired by the phenomenological model developed in Ref. [34], we have found that the conductance for the spin- $\sigma$ channel is

$$
G_{\sigma}(E, \phi)=G_{\sigma}^{\text {iso }}(E)+G_{\sigma}^{\text {aniso }}(E) \cos (2 \phi),
$$
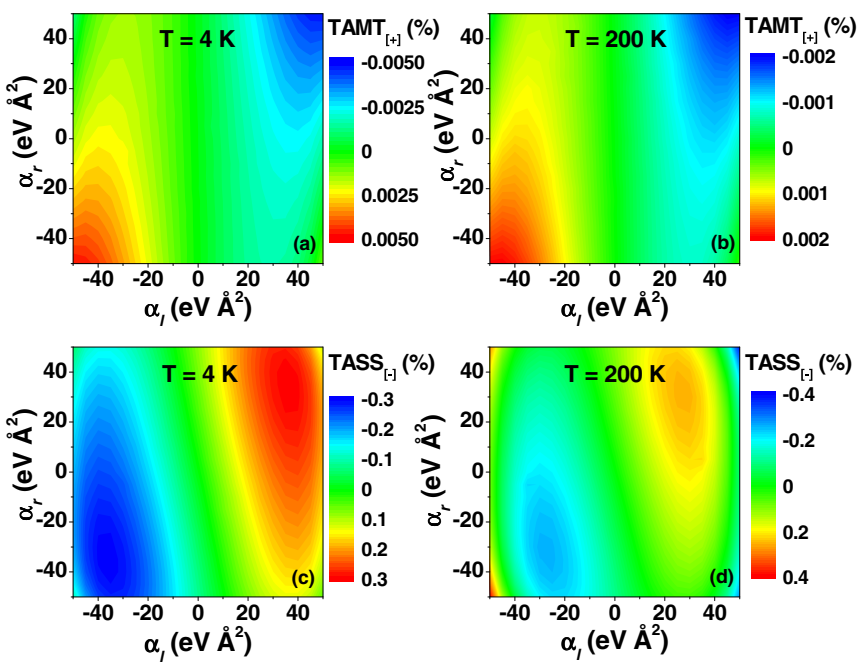

FIG. 8. (Color online) Same as in Fig. 7 but for the TAMT ${ }_{[+]}[(a)$ and (b)] and the TASS $[-][(\mathrm{c})$ and (d)]. where $G_{\sigma}^{\text {iso }}$ and $G_{\sigma}^{\text {aniso }}$ are phenomenological parameters [60] that satisfy that $G_{\sigma}^{\text {iso }} \gg G_{\sigma}^{\text {aniso }}$. For the special case where no SOI fields are present the anisotropy disappears, meaning $G_{\sigma}^{\text {aniso }}=0$.

Thereupon, taking advantage of Mott's relation for the Seebeck coefficient of the spin- $\sigma$ channel [see Appendix B Eq. (B5)] in Eqs. (6a), (6b), (7a), and (7b), respectively, and using Eq. (34) for $G$ and $G_{s}$, we find that the TAMT, TAMT ${ }_{[+]}$, $\operatorname{TASS}_{[-]}$, and TASS are all proportional to $(1-\cos 2 \phi)$. For each case, the corresponding amplitude is a specific function of $G_{\sigma}^{\text {iso }}$ and $G_{\sigma}^{\text {aniso }}$. Within the phenomenological approach these parameters cannot be computed, meaning that no quantitative predictions can be made. Nevertheless, this description is good enough in order to describe qualitatively the angular dependence found in Figs. 3 and 4. The quantitative results are obtained using the theory described in the previous section.

\section{SUMMARY}

We have studied thermally induced spin-dependent transport across a three-layer MTJ with a single ferromagnetic electrode in the presence of interfacial spin-orbit coupling. Prompted by previous works where TAMR was observed for $\mathrm{Fe} / \mathrm{GaAs} / \mathrm{Au}$ MTJs [26], we have shown that a similar anisotropy can be found in the (effective) thermopower and in the spin-dependent Seebeck and spin-Seebeck coefficients, when rotating the magnetization in the ferromagnetic lead with respect to a reference crystallographic axis. This anisotropy is due to the combined effect of the Bychkov-Rashba and the Dresselhaus spin-orbit fields, which posses a characteristic $C_{2 v}$ symmetry that appears in the TAMT, TAMT $_{[+]}, \mathrm{TASS}_{[-]}$, and TASS, as was the case for the TAMR. Finally, since the TAMT effect has recently been experimentally observed in an allsemiconductor MTJ [37], we believe that the TAMT, TAMT ${ }_{[+]}$, TASS $_{[-]}$, and TASS effects predicted in this work might also be measurable in similar experiments for $\mathrm{Fe} / \mathrm{GaAs} / \mathrm{Au}$ MTJs. Furthermore, inspired by recent experiments where anisotropic electric spin-injection for ferromagnetic-silicon interfaces has been observed [29], we hope this work may encourage the observation of anisotropic thermal spin injection.

\section{ACKNOWLEDGMENTS}

The authors are grateful for the financial support offered by the Deutsche Forschungsgemeinschaft (DFG) via the Priority Program "Spin Caloric Transport" (SPP 1538).

\section{APPENDIX A: LINEAR RESPONSE}

The Fermi-Dirac distribution is

$$
f_{L(R)}(E)=\frac{1}{1+e^{\left(E-\mu_{L(R)}\right) / k_{B} T_{L(R)}}},
$$

where $\mu_{L}=\mu_{R}+e V_{\text {bias }}, T_{L}=T_{R}+\Delta T, \mu_{L} / \mu_{R}$ and $T_{L} / T_{R}$ are the chemical potential and the temperature of the left/right electrode, respectively, and $V_{\text {bias }}$ and $\Delta T$ correspond to a bias voltage and a temperature gradient applied to the system, respectively. In the linear response regime,

$$
f_{L}-f_{R} \simeq\left(-\frac{\partial f_{0}}{\partial E}\right)\left(e V_{\text {bias }}+\frac{\Delta T}{T_{0}}\left(E-\mu_{0}\right)\right),
$$


where $\mu_{0}$ and $T_{0}$ are the equilibrium values, i.e., when $V_{\text {bias }}=0$ and $\Delta T=0$. Since

$$
S_{\sigma}=-\frac{1}{G_{\sigma}} \frac{I_{\sigma}}{\Delta T}
$$

[see Eq. (8)], taking $V_{\text {bias }}=0$ and replacing Eqs. (9) and (A2) in Eq. (A3) leads to Eq. (11) for the Seebeck coefficient for the spin- $\sigma$ channel.

\section{APPENDIX B: MOTT'S LAW}

Replacing Eq. (A2) in Eq. (9) yields

$$
I_{\sigma}=\frac{1}{e} \int K_{\sigma}(E)\left(-\frac{\partial f_{0}}{\partial E}\right) d E,
$$

where

$$
K_{\sigma}(E):=\left(V_{\text {bias }}+\frac{E-\mu_{0}}{e T_{0}} \Delta T\right) g_{\sigma}(E) .
$$

Using now the Sommerfeld expansion [46], we find that

$$
I_{\sigma}=K_{\sigma}\left(\mu_{0}\right)+\left.\sum_{n=1}^{\infty} a_{n}\left(k_{B} T_{0}\right)^{2 n} \frac{d^{2 n}}{d E^{2 n}} K_{\sigma}\right|_{E=\mu_{0}}
$$

where

$$
a_{n}=2\left(2^{2 n-1}-1\right) \frac{\pi^{2 n}}{(2 n) !} B_{n},
$$

and $B_{n}$ are the Bernoulli numbers. Taking $V_{\text {bias }}=0$ and $n=$ 1 (which is a good approximation for low temperatures) in Eq. (B3), and replacing it together with Eq. (B2) in Eq. (A3) we obtain

$$
S_{\sigma}=-\frac{k_{B}^{2}}{e} \frac{\pi^{2}}{3}\left(\left.\frac{d}{d E} \log g_{\sigma}(E)\right|_{E=\mu_{0}}\right) T_{0}
$$

This expression is the analog to Mott's law [58], but for the spin- $\sigma$ channel. Finally, replacing Eq. (B5) in Eq. (3) we arrive at

$$
S=-\frac{k_{B}^{2}}{e} \frac{\pi^{2}}{3}\left(\left.\frac{d}{d E} \log g(E)\right|_{E=\mu_{0}}\right) T_{0},
$$

which is nothing other than the well-known Mott relation for the thermopower [58], where $g=g_{\uparrow}+g_{\downarrow}$.

\section{APPENDIX C: RECTANGULAR POTENTIAL BARRIER MODEL}

The one-dimensional stationary Pauli-Schrödinger equation for the longitudinal modes is

$$
\left(\begin{array}{cc}
h(z) & s(z) \\
s^{*}(z) & h(z)
\end{array}\right)\left(\begin{array}{c}
\varphi_{\uparrow}(z) \\
\varphi_{\downarrow}(z)
\end{array}\right)=E\left(\begin{array}{c}
\varphi_{\uparrow}(z) \\
\varphi_{\downarrow}(z)
\end{array}\right),
$$

where

$$
h(z)=\frac{\hbar^{2} k_{\|}^{2}}{2 m(z)}+p_{z}\left(\frac{1}{2 m(z)} p_{z}\right)+V_{0}\left[\theta\left(z-z_{l}\right)-\theta\left(z-z_{r}\right)\right],
$$

$$
\begin{aligned}
s(z)= & -\frac{\Delta}{2} e^{-i \theta} \theta\left(-z+z_{l}\right)-i k_{\|} \alpha e^{-i \theta_{\|}} \delta\left(z-z_{l}\right) \\
& -\frac{\gamma(z)}{\hbar^{2}} k_{\|} e^{i \theta_{\|}} p_{z}^{2}+\sum_{j=l, r} \delta\left(z-z_{j}\right)\left(\delta_{j l}-\delta_{j r}\right)\left(\frac{\gamma}{\hbar}\right) \\
& \times i k_{\|} e^{i \theta_{\|}} p_{z},
\end{aligned}
$$

where $\theta_{\|}=\arg \left(k_{y} / k_{x}\right)$. Note that, for simplicity, we have taken $\alpha_{r}=0$ and $\alpha_{l}=\alpha$. The stationary solutions of Eq. (C1) in the electrodes are the incident and transmitted wave functions given in Eqs. (19a) and (19b), respectively, and the reflected wave function:

$$
\boldsymbol{\varphi}_{r}(z)=r_{\sigma, \sigma} e^{-i k_{1 \sigma} z} \boldsymbol{v}_{\sigma}+r_{\bar{\sigma}, \sigma} e^{-i k_{1 \bar{\sigma}} z} \boldsymbol{v}_{\bar{\sigma}},
$$

where $k_{1 \sigma}$ and $\boldsymbol{v}_{\sigma}$ are given by Eqs. (20a) and (21), respectively, and $r_{\sigma, \sigma}$ and $r_{\bar{\sigma}, \sigma}$ are the reflection probability amplitudes, analogous to the transmission probability amplitudes discussed in the main text. Furthermore, the solution of Eq. (C1) in the tunnel barrier is

$$
\boldsymbol{\varphi}_{2}(z)=\sum_{i= \pm}\left(C_{\sigma, i} e^{k_{2 i} z}+D_{\sigma, i} e^{-k_{2 i} z}\right) \boldsymbol{v}_{2 i},
$$

where

$$
k_{2 \pm}=\frac{1}{\sqrt{1 \mp \frac{2 m_{2} \gamma k_{\|}}{\hbar^{2}}}} \sqrt{\frac{2 m_{2}}{\hbar^{2}}\left(V_{0}-E\right)+k_{\|}^{2}}
$$

and

$$
\boldsymbol{v}_{2 \pm}=\frac{1}{\sqrt{2}}\left(\begin{array}{c}
1 \\
\pm e^{-i \theta_{\|}}
\end{array}\right) .
$$

Finally, boundary conditions are imposed on the wave functions and their derivatives for computing the reflection and the transmission probability amplitudes. First, the wave functions must be continuous at the interfaces

$$
\begin{aligned}
& \varphi_{1}\left(z_{l}\right)=\varphi_{2}\left(z_{l}\right), \\
& \varphi_{2}\left(z_{r}\right)=\varphi_{3}\left(z_{r}\right) .
\end{aligned}
$$

And second, the derivatives of the wave functions must satisfy the following equations:

$$
\begin{aligned}
& \frac{\hbar^{2}}{2}\left(\left.\frac{1}{m_{1}} \frac{\partial \varphi_{1 \uparrow}}{\partial z}\right|_{z=z_{l}}-\left.\frac{1}{m_{2}} \frac{\partial \varphi_{2 \uparrow}}{\partial z}\right|_{z=z_{l}}\right)-i k_{\|} \alpha e^{-i \theta_{\|}} \varphi_{1 \downarrow}\left(z_{l}\right)+\left.k_{\|} \gamma e^{i \theta_{\|}} \frac{\partial \varphi_{2 \downarrow}}{\partial z}\right|_{z=z_{l}}=0, \\
& \frac{\hbar^{2}}{2}\left(\left.\frac{1}{m_{1}} \frac{\partial \varphi_{1 \downarrow}}{\partial z}\right|_{z=z_{l}}-\left.\frac{1}{m_{2}} \frac{\partial \varphi_{2 \downarrow}}{\partial z}\right|_{z=z_{l}}\right)+i k_{\|} \alpha e^{i \theta_{\|}} \varphi_{1 \uparrow}\left(z_{l}\right)+\left.k_{\|} \gamma e^{-i \theta_{\|}} \frac{\partial \varphi_{2 \uparrow}}{\partial z}\right|_{z=z_{l}}=0,
\end{aligned}
$$


for the left barrier, and

$$
\begin{aligned}
& \frac{\hbar^{2}}{2}\left(\left.\frac{1}{m_{2}} \frac{\partial \varphi_{2 \uparrow}}{\partial z}\right|_{z=z_{r}}-\left.\frac{1}{m_{3}} \frac{\partial \varphi_{3 \uparrow}}{\partial z}\right|_{z=z_{r}}\right)-\left.k_{\|} \gamma e^{i \theta_{\|}} \frac{\partial \varphi_{2 \downarrow}}{\partial z}\right|_{z=z_{r}}=0, \\
& \frac{\hbar^{2}}{2}\left(\left.\frac{1}{m_{2}} \frac{\partial \varphi_{2 \downarrow}}{\partial z}\right|_{z=z_{r}}-\left.\frac{1}{m_{3}} \frac{\partial \varphi_{3 \downarrow}}{\partial z}\right|_{z=z_{r}}\right)-\left.k_{\|} \gamma e^{-i \theta_{\|}} \frac{\partial \varphi_{2 \uparrow}}{\partial z}\right|_{z=z_{r}}=0,
\end{aligned}
$$

for the right barrier. The first and second terms on the left-hand side of these equations are the so called BenDaniel-Duke boundary conditions [59], which correspond to the generalization for heterostructures of the requirement that the derivatives of the wave functions should also be continuous at the interfaces. The third and fourth terms in Eqs. (C9a) and (C9b) are due to the BR-SOI and D-SOI at the Fe/GaAs interface, respectively. And the third term in Eqs. (C10a) and (C10b) is due to the D-SOI at the GaAs/Au interface.

The exact expressions for the transmission amplitudes $t_{\sigma, \sigma}$ and $t_{\sigma, \bar{\sigma}}$ are quite lengthy. However, a simplified analytical expressions for $t_{\sigma, \sigma}$ and $t_{\sigma, \bar{\sigma}}$ can be obtained in the limit $k_{2 \pm} d \gg 1$. In such case, one finds the following approximate relations for the tunneling amplitudes:

$$
\begin{aligned}
& t_{\sigma, \sigma}=-\frac{D_{\sigma, \sigma}}{D}, \\
& t_{\sigma, \bar{\sigma}}=-\frac{D_{\sigma, \bar{\sigma}}}{D},
\end{aligned}
$$

where $D=f_{-}(-) f_{+}(-)-f_{-}(+) f_{+}(+)$, with

$$
f_{ \pm}(\lambda)=\frac{d}{2}\left(\frac{m_{0}}{m_{ \pm \lambda}} k_{2 \mp \lambda}-i k_{3}\right)\left(1-\lambda \sigma e^{i\left(\theta-\theta_{\|}\right)}\right)
$$

and

$$
\frac{1}{m_{ \pm}}=\frac{1}{m_{2}}\left(1 \pm \frac{2 m_{2} \gamma k_{\|}}{\hbar^{2}}\right) .
$$

Furthermore, we have

$$
\begin{aligned}
& D_{\sigma, \sigma}=\frac{2 m_{0} d}{m_{+}} k_{2-} f_{-}(+) g_{-}-\frac{2 m_{0} d}{m_{-}} k_{2+} f_{-}(-) g_{+}, \\
& D_{\sigma, \bar{\sigma}}=\frac{2 m_{0} d}{m_{+}} k_{2-} f_{+}(-) g_{-}-\frac{2 m_{0} d}{m_{-}} k_{2+} f_{+}(+) g_{+} .
\end{aligned}
$$

In these equations we introduced the notation

$$
g_{ \pm}=\frac{i d \sqrt{k_{1 \sigma}}\left[\left(f_{0} \mp h_{1}-\frac{m_{0} d}{m_{ \pm}} k_{2 \mp}\right)\left(1 \pm \sigma e^{i\left(\theta-\theta_{\|}\right)}\right) \mp h_{2}\left(1 \mp \sigma e^{i\left(\theta-\theta_{\|}\right)}\right)\right] e^{-k_{2 \pm} d}}{h_{2}^{2}+\left(f_{0}-h_{1}-\frac{m_{0} d}{m_{+}} k_{2-}\right)\left(f_{0}+h_{1}-\frac{m_{0} d}{m_{-}} k_{2+}\right)},
$$

where $f_{0}=i\left(k_{1 \sigma}+k_{1 \bar{\sigma}}\right) d / 2$ and

$$
\begin{aligned}
& h_{1}=\frac{i \sigma d}{2}\left(k_{1 \sigma}-k_{1 \bar{\sigma}}\right) \cos \left(\theta-\theta_{\|}\right)-\frac{\alpha k_{\|} Q}{V_{0}} \sin \left(2 \theta_{\|}\right), \\
& h_{2}=-\frac{\sigma d}{2}\left(k_{1 \sigma}-k_{1 \bar{\sigma}}\right) \sin \left(\theta-\theta_{\|}\right)-i \frac{\alpha k_{\|} Q}{V_{0}} \cos \left(2 \theta_{\|}\right),
\end{aligned}
$$

where $Q=2 m_{0} V_{0} d / \hbar^{2}$. Note that the effective mass in the metallic leads has been taken as the bare electron mass (i.e., $m_{1}=m_{3}=m_{0}$ ). The obtained approximate expressions for the tunneling coefficients are valid up to first order in $\exp \left(-k_{2 \pm} d\right)$. This approximation is therefore appropriate for treating junctions with high and not too thin potential barriers. Taking the Fermi energy as the zero of the energy scale, the height of the barrier is about $V_{0} \approx 0.75 \mathrm{eV}$.

\section{APPENDIX D: DIRAC-DELTA FUNCTION BARRIER MODEL}

The discussion of this model is analogous to the discussion of the rectangular barrier model carried out in the previous section. Therefore, onwards we only highlight the differences between both models. First, Eqs. (C2a) and (C2b) become

$$
\begin{aligned}
& h(z)=\frac{\hbar^{2} k_{\|}^{2}}{2 m(z)}+p_{z}\left(\frac{1}{2 m(z)} p_{z}\right)+V_{0} d \delta\left(z-z_{2}\right), \quad \text { (D1a) } \\
& s(z)=-\frac{\Delta}{2} e^{-i \theta} \theta\left(-z+z_{l}\right)-k_{\|}\left(i \alpha e^{-i \theta_{\|}}-\bar{\gamma} e^{i \theta_{\|}}\right) \delta\left(z-z_{2}\right),
\end{aligned}
$$

the solutions of which in the electrodes are the same as in the rectangular barrier model. Second, the boundary conditions become

$$
\varphi_{1}\left(z_{2}\right)=\varphi_{3}\left(z_{2}\right),
$$


and

$$
\begin{aligned}
& \frac{\hbar^{2}}{2}\left(\left.\frac{1}{m_{1}} \frac{\partial \varphi_{1 \uparrow}}{\partial z}\right|_{z=z_{2}}-\left.\frac{1}{m_{3}} \frac{\partial \varphi_{3 \uparrow}}{\partial z}\right|_{z=z_{2}}\right) \\
& \quad+V_{0} d \varphi_{1 \uparrow}\left(z_{2}\right)-\left[k_{\|}\left(i \alpha e^{-i \theta_{\|}}-\bar{\gamma} e^{i \theta_{\|}}\right)\right] \varphi_{1 \downarrow}\left(z_{2}\right)=0 \\
& \frac{\hbar^{2}}{2}\left(\left.\frac{1}{m_{1}} \frac{\partial \varphi_{1 \downarrow}}{\partial z}\right|_{z=z_{2}}-\left.\frac{1}{m_{3}} \frac{\partial \varphi_{3 \downarrow}}{\partial z}\right|_{z=z_{2}}\right) \\
& \quad+V_{0} d \varphi_{1 \downarrow}\left(z_{2}\right)+\left[k_{\|}\left(i \alpha e^{i \theta_{\|}}+\bar{\gamma} e^{-i \theta_{\|}}\right)\right] \varphi_{1 \uparrow}\left(z_{2}\right)=0 .
\end{aligned}
$$$$
\text { (D3) }
$$

(D4)

And finally, the transmission amplitudes are

$$
t_{\sigma, \sigma}=-\frac{8 d^{2} \sqrt{k_{1 \sigma}}\left(k_{1 \bar{\sigma}}+k_{3}+i Q\right)}{\Omega}+\frac{8 i d \sqrt{k_{1 \sigma}}\left(\mathbf{U} \cdot \mathbf{S}_{\sigma, \sigma}\right)}{\Omega}
$$

$$
t_{\sigma, \bar{\sigma}}=-\frac{8 i d \sqrt{k_{1 \sigma}}\left(\mathbf{U} \cdot \mathbf{S}_{\sigma, \bar{\sigma}}\right)}{\Omega},
$$

where

$$
\Omega=\Omega_{+}(-) \Omega_{-}(+)-\Omega_{+}(+) \Omega_{-}(-),
$$

with

$$
\Omega_{ \pm}(\lambda)=d\left(k_{1, \pm \sigma}+k_{3}+i Q\right)(1 \pm \lambda)+2 i\left(\mathbf{U} \cdot \mathbf{S}_{ \pm \sigma, \lambda \sigma}\right),
$$

and $\lambda= \pm 1$. The vectors $\mathbf{S}_{\sigma, \pm \sigma}$ are given by

$$
\mathbf{S}_{\sigma, \pm \sigma}=\boldsymbol{v}_{\sigma}^{\dagger} \boldsymbol{\sigma} \boldsymbol{v}_{ \pm \sigma}
$$

where $\mathbf{U}=\left(2 m_{0} d / \hbar^{2}\right) \mathbf{w}$ with

$$
\mathbf{w}=\left(-\alpha k_{y}+\bar{\gamma} k_{x}, \alpha k_{x}-\bar{\gamma} k_{y}, 0\right)
$$

[1] R. Barnard, Thermoelectricity in Metals and Alloys (Taylor \& Francis, London, 1972).

[2] J. Ziman, Electrons and Phonons: The Theory of Transport Phenomena in Solids, Oxford Classic Texts in the Physical Sciences (Clarendon Press, Oxford, 2001).

[3] M. Johnson and R. H. Silsbee, Phys. Rev. B 35, 4959 (1987).

[4] I. Žutić, J. Fabian, and S. Das Sarma, Rev. Mod. Phys. 76, 323 (2004).

[5] J. Fabian, A. Matos-Abiague, C. Ertler, P. Stano, and I. Žutić, Acta Physica Slovaca: Reviews and Tutorials 57, 565 (2007).

[6] K. Uchida, S. Takahashi, K. Harii, J. Ieda, W. Koshibae, K. Ando, S. Maekawa, and E. Saitoh, Nature (London) 455, 778 (2008).

[7] K. Uchida, J. Xiao, H. Adachi, J. Ohe, S. Takahashi, J. Ieda, T. Ota, Y. Kajiwara, H. Umezawa, H. Kawai et al., Nat. Mater. 9, 894 (2010).

[8] C. M. Jaworski, J. Yang, S. Mack, D. D. Awschalom, J. P. Heremans, and R. C. Myers, Nat. Mater. 9, 898 (2010).

[9] G. E. Bauer, A. H. MacDonald, and S. Maekawa, Solid State Commun. 150, 459 (2010).

[10] G. E. W. Bauer, E. Saitoh, and B. J. van Wees, Nat. Mater. 11, 391 (2012).

[11] M. Walter, J. Walowski, V. Zbarsky, M. Münzenberg, M. Schfers, D. Ebke, G. Reiss, A. Thomas, P. Peretzki, M. Seibt et al., Nat. Mater. 10, 742 (2011).

[12] J.-C. Le Breton, S. Sharma, H. Saito, S. Yuasa, and R. Jansen, Nature (London) 475, 82 (2011).

[13] N. Liebing, S. Serrano-Guisan, K. Rott, G. Reiss, J. Langer, B. Ocker, and H. W. Schumacher, Phys. Rev. Lett. 107, 177201 (2011).

[14] W. Lin, M. Hehn, L. Chaput, B. Negulescu, S. Andrieu, F. Montaigne, and S. Mangin, Nat. Commun. 3, 744 (2012).

[15] J. Flipse, F. Bakker, A. Slachter, F. Dejene, and B. van Wees, Nat. Nanotechnol. 7, 166 (2012).

[16] K.-R. Jeon, B.-C. Min, S.-Y. Park, K.-D. Lee, H.-S. Song, Y.-H. Park, Y.-H. Jo, and S.-C. Shin, Sci. Rep. 2, 962 (2012).
[17] Y. Ni, K. Yao, H. Fu, G. Gao, S. Zhu, and S. Wang, Sci. Rep. 3, 1380 (2013).

[18] I. J. Vera-Marun, B. J. van Wees, and R. Jansen, Phys. Rev. Lett. 112, 056602 (2014).

[19] M. Tanaka and Y. Higo, Phys. Rev. Lett. 87, 026602 (2001).

[20] Y. Higo, H. Shimizu, and M. Tanaka, J. Appl. Phys. 89, 6745 (2001).

[21] D. Chiba, F. Matsukura, and H. Ohno, Physica E 21, 966 (2004).

[22] C. Rüster, C. Gould, T. Jungwirth, J. Sinova, G. M. Schott, R. Giraud, K. Brunner, G. Schmidt, and L. W. Molenkamp, Phys. Rev. Lett. 94, 027203 (2005).

[23] H. Saito, S. Yuasa, and K. Ando, Phys. Rev. Lett. 95, 086604 (2005).

[24] L. Gao, X. Jiang, S.-H. Yang, J. D. Burton, E. Y. Tsymbal, and S. S. P. Parkin, Phys. Rev. Lett. 99, 226602 (2007).

[25] C. Gould, C. Rüster, T. Jungwirth, E. Girgis, G. M. Schott, R. Giraud, K. Brunner, G. Schmidt, and L. W. Molenkamp, Phys. Rev. Lett. 93, 117203 (2004).

[26] J. Moser, A. Matos-Abiague, D. Schuh, W. Wegscheider, J. Fabian, and D. Weiss, Phys. Rev. Lett. 99, 056601 (2007).

[27] R. S. Liu, L. Michalak, C. M. Canali, L. Samuelson, and H. Pettersson, Nano Lett. 8, 848 (2008).

[28] N. Néel, S. Schröder, N. Ruppelt, P. Ferriani, J. Kröger, R. Berndt, and S. Heinze, Phys. Rev. Lett. 110, 037202 (2013).

[29] S. Sharma, S. P. Dash, H. Saito, S. Yuasa, B. J. van Wees, and R. Jansen, Phys. Rev. B 86, 165308 (2012).

[30] L. Brey, C. Tejedor, and J. Fernández-Rossier, Appl. Phys. Lett. 85, 1996 (2004).

[31] P. Sankowski, P. Kacman, J. A. Majewski, and T. Dietl, Phys. Rev. B 75, 045306 (2007).

[32] M. N. Khan, J. Henk, and P. Bruno, J. Phys.: Condens. Matter 20, 155208 (2008).

[33] A. N. Chantis, K. D. Belashchenko, E. Y. Tsymbal, and M. van Schilfgaarde, Phys. Rev. Lett. 98, 046601 (2007).

[34] A. Matos-Abiague and J. Fabian, Phys. Rev. B 79, 155303 (2009). 
[35] A. Matos-Abiague, M. Gmitra, and J. Fabian, Phys. Rev. B 80, 045312 (2009).

[36] M. Wimmer, M. Lobenhofer, J. Moser, A. Matos-Abiague, D. Schuh, W. Wegscheider, J. Fabian, K. Richter, and D. Weiss, Phys. Rev. B 80, 121301 (2009).

[37] T. Naydenova, P. Dürrenfeld, K. Tavakoli, N. Pégard, L. Ebel, K. Pappert, K. Brunner, C. Gould, and L. W. Molenkamp, Phys. Rev. Lett. 107, 197201 (2011).

[38] C. Jia and J. Berakdar, Appl. Phys. Lett. 98, 192111 (2011).

[39] V. Popescu and P. Kratzer, Phys. Rev. B 88, 104425 (2013).

[40] S. Wimmer, D. Ködderitzsch, and H. Ebert, Phys. Rev. B 89, 161101 (2014).

[41] L. Gravier, S. Serrano-Guisan, F. Reuse, and J.-P. Ansermet, Phys. Rev. B 73, 024419 (2006).

[42] R. Jansen, A. M. Deac, H. Saito, and S. Yuasa, Phys. Rev. B 85, 094401 (2012).

[43] A. Slachter, F. L. Bakker, J.-P. Adam, and B. J. van Wees, Nat. Phys. 6, 879 (2010).

[44] M. Czerner, M. Bachmann, and C. Heiliger, Phys. Rev. B 83, 132405 (2011).

[45] B. Scharf, A. Matos-Abiague, I. Žutić, and J. Fabian, Phys. Rev. B 85, 085208 (2012).

[46] N. W. Ashcroft and N. D. Mermin, Solid State Physics (Brooks/Cole, Pacific Blue, CA, 1976).

[47] M. Gmitra, A. Matos-Abiague, C. Draxl, and J. Fabian, Phys. Rev. Lett. 111, 036603 (2013).
[48] S. Putz, M. Gmitra, and J. Fabian, Phys. Rev. B 90, 045315 (2014).

[49] C. López-Monís, A. Matos-Abiague, and J. Fabian, Phys. Rev. B 89, 054419 (2014).

[50] J. H. Davies, The Physics of Low-dimensional Semiconductors: An Introduction (Cambridge University Press, Cambridge, 1998).

[51] J. C. Slonczewski, Phys. Rev. B 39, 6995 (1989).

[52] C. H. Shang, J. Nowak, R. Jansen, and J. S. Moodera, Phys. Rev. B 58, R2917(R) (1998).

[53] T. Hagler, R. Kinder, and G. Bayreuther, J. Appl. Phys. 89, 7570 (2001).

[54] M. Lobenhofer, Ph.D. thesis, University of Regensburg, 2013.

[55] Y. P. Varshni, Physica 34, 149 (1967).

[56] P. Lautenschlager, M. Garriga, S. Logothetidis, and M. Cardona, Phys. Rev. B 35, 9174 (1987).

[57] P. S. Eldridge, W. J. H. Leyland, P. G. Lagoudakis, O. Z. Karimov, M. Henini, D. Taylor, R. T. Phillips, and R. T. Harley, Phys. Rev. B 77, 125344 (2008).

[58] N. Mott and H. Jones, The Theory of the Properties of Metals and Alloys, Dover Books on Relativity and Related Areas (Dover Publications, Inc., New York, 1958).

[59] D. J. BenDaniel and C. B. Duke, Phys. Rev. 152, 683 (1966).

[60] The difference between Eq. (34) and the conductance found in Ref. [34] is that here it is spin and energy dependent. Apart from this, the derivation is the same. $G_{\sigma}^{\text {iso }}$ and $G_{\sigma}^{\text {aniso }}$ are given by Eqs. (52) and (54) of Ref. [34], respectively. 